\title{
Biodegradation of pharmaceuticals by Rhodococcus rhodochrous and Aspergillus niger by co-metabolism
}

\author{
Hervé Gauthier, Viviane Yargeau*, David G. Cooper \\ Department of Chemical Engineering, McGill University, 3610 University, Montreal, Canada H3A 2B2
}

\section{A R T I C L E I N F O}

\section{Article history:}

Received 7 August 2009

Received in revised form 30 November 2009

Accepted 6 December 2009

Available online 20 January 2010

\section{Keywords:}

Biodegradation

Carbamazepine

Metoprolol

Sulfamethizole

Sulfamethoxazole

Trimethoprim

\begin{abstract}
A B S T R A C T
This work investigated the possible fate of pharmaceuticals in the environment that are known to be resistant to biodegradation. A co-metabolism approach, adding a readily degradable carbon source, was used to study the biodegradation of some pharmaceuticals. The pharmaceuticals selected were all known to be micro pollutants and frequently used by humans. The microorganisms used primarily were Rhodococcus rhodochrous, known to co-metabolize difficult to degrade hydrocarbons and Aspergillus niger. Because of the long periods of time required for the degradation experiments after growth had reached the stationary phase, it was found to be necessary to correct for water loss from the media. Co-metabolism of carbamazepine, sulfamethizole and sulfamethoxazole was observed and as much as $20 \%$ of these compounds could be removed. Small amounts of stable metabolites were observed during the degradation of some of these drugs and these were different from the metabolites obtained from abiotic degradation. A metabolite arising from the biodegradation of sulfamethoxazole by $R$. rhodochrous was identified.
\end{abstract}

(C) 2009 Elsevier B.V. All rights reserved.

\section{Introduction}

Pharmaceuticals include a wide range of chemicals with very different structures, functions, behaviours and activities. In fact, more than 3000 active ingredients have been approved in Europe just for human medical care (Zwiener, 2007). Most of the pharmaceuticals produced ultimately make their way into the environment affecting the flora and fauna to different extents. The presence of pharmaceutical residues in the environment was reported for the first time in the late 1970s (Jones et al., 2005). Passage through a wastewater treatment plant does not guarantee the removal of pharmaceuticals. Many studies showed the inefficiency of wastewater treatment plants for the removal of pharmaceuticals (Ternes, 1998). Ternes reported that the removal efficiency for 32 pharmaceutical residues after passage through a wastewater treatment unit ranged from 7 to $96 \%$. The removal process is inadequate for many pharmaceuticals simply because wastewater treatment units have not been designed to remove such compounds (Kolpin et al., 2002).

The usual reported concentrations of pharmaceuticals in water bodies are in the magnitude of $\mathrm{ng} / \mathrm{L}$ or low $\mu \mathrm{g} / \mathrm{L}$ and rarely exceed the drinking-water-guidelines (Kolpin et al., 2002). Most of these pharmaceuticals have not been regulated yet and scientists are concerned about the potential negative effects of these compounds on ecosystems and public health.

\footnotetext{
* Corresponding author. Tel.: +1 514398 2273; fax: +1 5143986678. E-mail address: viviane.yargeau@mcgill.ca (V. Yargeau).
}

The concentration of pharmaceuticals in tap water is in the order of ng per litre, which is much lower than the doses prescribed in therapeutic treatment. However, a few studies have predicted the likelihood of profound effects caused by pharmaceuticals with highly specific mechanisms, even at these extremely low concentrations (Daughton and Ternes, 1999). Possible effects of long term exposure to pharmaceuticals include abnormal physiological processes and reproductive impairment (Jobling et al., 1998), increased incidences of cancer (Davis and Bradlow, 1995) and the potential for increased toxicity in chemical mixtures (Kolpin et al., 2002).

Most of the studies on biodegradation of pharmaceuticals looked at the removal of these compounds by activated sludge (Oppenheimer et al., 2007; Stackelberg et al., 2007). Activated sludge is a consortium of fast growing microorganisms in a medium with high carbon source concentration, which is not representative of the bacterial population and conditions observed in natural environmental systems. Pure cultures offer the possibility for microorganisms with oligotrophic metabolism to achieve biodegradation of xenobiotics and offer a way to study the environmental fate of pharmaceuticals.

The aim of this research was to study the biodegradation of common pharmaceuticals by relevant microorganisms that were growing rapidly on a more easily used carbon source than the pharmaceutical. The pharmaceuticals selected were all known to be resistant to biodegradation, which limits the amount of biomass that can be generated. However, in most environmental situations, the various pharmaceuticals would be present in concentrations lower than other available carbon sources. This means that it was reasonable to add a second, more easily utilized carbon source and still produce 
results relevant to the actual situation in the environment. By adding this second carbon source, enough biomass was generated to act on the target compound. This had a secondary advantage as it meant that the studies could be done with low concentrations of pharmaceuticals to decrease the risk of toxic effects.

Similar studies with plasticizers commonly found in the environment showed significant enhancement of biodegradation of these compounds by organisms growing on several different types of carbon (Kermanshahi-Pour et al., 2009; Nalli et al., 2006a,b). As well, this type of interaction led to the accumulation of plasticizer metabolites that were resistant to further degradation. A recent study has shown that the hormone 17- $\alpha$ ethinylestradiol was much more easily biodegraded when another carbon source was added to the medium (O'Grady and Yargeau, 2009).

The pharmaceuticals studied have been repeatedly found in the environment (Åke and Bo, 2005; Kolpin, et al., 2002; Tixier, et al., 2003; Yargeau et al., 2007) and are considered to be micro pollutants. These include three antibiotics: sulfamethoxazole, sulfamethizole and trimethoprim, as well as one antiepileptic drug; carbamazepine, and one hypertension medicine; metoprolol. Pure cultures of microorganisms commonly found in soil were chosen to evaluate the biodegradability of the selected drugs. The microorganisms chosen were known to degrade similar molecules as the pharmaceuticals of interest or at least act on the same functional groups. The primary microorganism used in these experiments was Rhodococcus rhodochrous. The previous work had shown that this bacterium was very efficient at partially metabolizing various difficult to degrade plasticizers, which are often observed in the environment (Kermanshahi-Pour et al., 2009; Nalli et al., 2006a,b).

\section{Materials and methods}

\subsection{Chemicals and microorganisms}

Carbamazepine (CBZ), sulfamethizole (SMZ), sulfamethoxazole (SMX), trimethoprim (TMP), metroprolol (MTP), dimethyl sulfoxide (DMSO), formic acid and ammonium acetate were purchased from Sigma-Aldrich. Methanol HPLC grade, acetonitrile HPLC grade, water HPLC grade, phosphoric acid HPLC grade, ethylenediaminetetraacetic acid dissodium salt $\left(\mathrm{Na}_{2} \mathrm{EDTA}\right)$, ammonium nitrate, dissodium hydrogen phosphate $\left(\mathrm{Na}_{2} \mathrm{HPO}_{4}\right)$ and monobasic potassium phosphate $\left(\mathrm{KH}_{2} \mathrm{PO}_{4}\right)$ were obtained from Fisher. Calcium chloride dihydrate and ferrous sulfate heptahydrate were bought from Acros whereas magnesium sulfate heptahydrate and D-glucose anhydrous were bought from A\&C. BHI and yeast extract were purchased from Becton, Dickinson and Company. The microorganisms were all purchased from ATCC and included R. rhodochrous (13808), Pseudomonas putida (12633), Pseudomonas fluorescens (13525), Bacillus subtilis (6051), Aspergillus niger (16888) and Sphingomonas herbicidovorans (700291).

\subsection{Growth experiments}

Growth experiments were conducted in shake flasks $(500 \mathrm{~mL})$ with a foam plug and placed in an incubator shaker at $26{ }^{\circ} \mathrm{C}$ and at $150 \mathrm{rpm}$. Each flask contained $100 \mathrm{~mL}$ of media and was autoclaved prior to inoculation. For experiments conducted with sulfamethizole, sulfamethoxazole, metoprolol and trimethoprim, shake flasks were covered with aluminium foil to prevent photodegradation of the compounds. For all experiments, microorganisms were transferred once in brain heart infusion (BHI) media, and then transferred twice in minimum mineral salt media (MMSM). The microorganisms were then grown in MMSM containing a pharmaceutical (acclimation period) and, finally, used to inoculate flasks for the degradation experiments. Three controls were used: with drug but without microorganisms; with dead microorganisms and without the drug. The first type of control was done to account for the abiotic degradation of the drug. The second type was done to account for any adsorption to the biomass and the last type of control was done to distinguish metabolites arising from the breakdown of the drug studied from metabolites resulting from the base metabolism of the microorganism. The flasks were weighed to allow calculations to correct the changes in pharmaceutical concentrations due to water evaporation.

Brain heart infusion media were prepared by weighing $3.7 \mathrm{~g}$ of BHI in a shake flask with $100 \mathrm{~mL}$ of distilled water. Minimum mineral salt media were prepared to have a final concentration of $0.018 \mathrm{~g} / \mathrm{L}$ of $\mathrm{Na}_{2}$ EDTA, $0.013 \mathrm{~g} / \mathrm{L}$ of ferrous sulfate hexahygrate, $0.013 \mathrm{~g} / \mathrm{L}$ of calcium chloride dihydrate, $0.25 \mathrm{~g} / \mathrm{L}$ of magnesium sulfate heptahydrate, $7.5 \mathrm{~g} / \mathrm{L}$ of dissodium hydrogen phosphate, $5 \mathrm{~g} / \mathrm{L}$ of monobasique potassium phosphate, $5 \mathrm{~g} / \mathrm{L}$ of ammonium nitrate and $0.5 \mathrm{~g} / \mathrm{L}$ of yeast extract. Glucose was added separately to achieve an initial concentration of $3 \mathrm{~g} / \mathrm{L}$. The glucose was not replenished during the experiment.

Inoculations were done in the laminar flow hood using sterilized equipment. In all experiments, $80 \mathrm{~mL}$ of the MMSM solution was added first. For CBZ, SMX and SMZ experiments, $10 \mathrm{~mL}$ of the stock solution and $10 \mathrm{~mL}$ of glucose solution were then added to each flask along with $1 \mathrm{~mL}$ of acclimated microorganisms. For TMP experiments, $1 \mathrm{~mL}$ of the stock solution, $1 \mathrm{~mL}$ of acclimated microorganisms and $10 \mathrm{~mL}$ of glucose solution were added to each flask. For controls without drugs, the stock solution was replaced by the same amount of distilled water. In controls without biomass, distilled water replaced the inoculum. The control with dead microorganisms consisted of acclimated biomass grown on MMSM without the drug and then autoclaved. The corresponding quantity of pharmaceutical was then added and the concentration monitored.

Each pharmaceutical was studied separately. The stock solution of carbamazepine was prepared at $100 \mathrm{ppm}$ in distilled water and was autoclaved prior to inoculation as no degradation of carbamazepine was observed during autoclaving. All of the others were subjected to filter sterilization since they were suspected to be heat sensitive. Sulfamethoxazole and sulfamethizole stock solutions were prepared at $400 \mathrm{ppm}$ in distilled water. Metroprolol stock solution was prepared at $1000 \mathrm{ppm}$ in distilled water. Trimethoprim stock solution was prepared at about 12,500 ppm in DMSO and kept under nitrogen. Sterile filters were used to transfer the solutions into flasks.

\subsection{Sampling procedure and corrections for water loss}

Samples were continued to be taken as long as changes in concentration were observed. Each flask was weighed at the beginning of the experiment and then before and after each sample $(2 \mathrm{~mL})$ was collected. All samples were removed in a laminar flow hood. Samples were centrifuged (IEC Thermo, model Micromax) for $10 \mathrm{~min}$ at $10,000 \mathrm{rpm}$. The supernatant was then filtered with $0.45 \mu \mathrm{m}$ PVDF filters (Fisher, cat \# 097203) at which point the samples were ready for HPLC analysis.

The values for concentration obtained from HPLC were corrected for water loss. The normalized concentration $\left(C_{N}(t)\right)$ in ppm was defined by the following equation:

$C_{\mathrm{N}}(t)=\frac{C_{\mathrm{M}}(t) * V(t)}{V_{\mathrm{N}}(t)}$

Where $C_{\mathrm{M}}(t)$ is the concentration measured by HPLC, $V(t)$ is the volume in the flask at time $(t)$ and $V_{\mathrm{N}}(t)$ is the normalized volume, as defined by Eqs. (2)-(4). The density was assumed to always be $1 \mathrm{~g} / \mathrm{mL}$ as all of the samples were mostly water.

$V(t)=\frac{\text { mass of flask }(t)_{\text {before sampling }}-\text { mass of emptyflask }(t=0)}{\rho}$ 


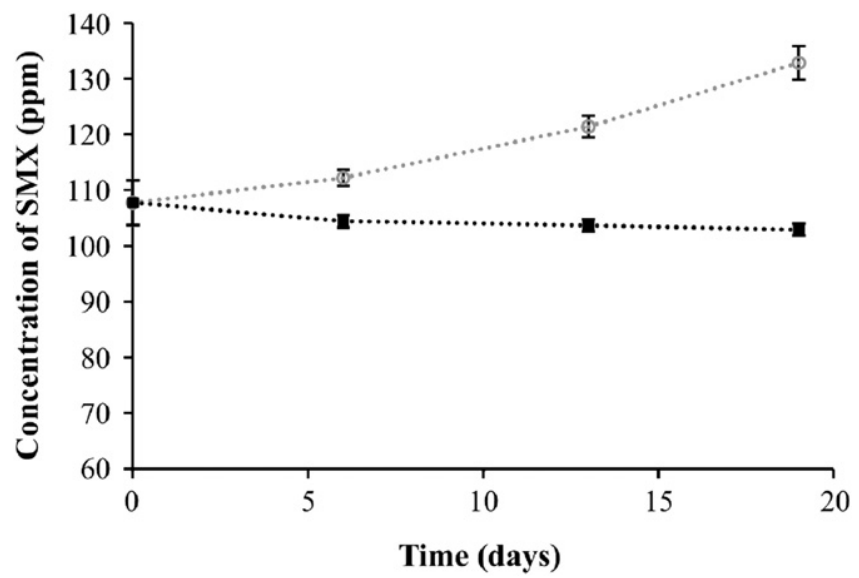

Fig. 1. Concentration of metoprolol in flasks containing Rhodococcus rhodochrouscorrected data.

$V_{\mathrm{N}}(t)=V(t)+\sum_{0}^{t} V_{\mathrm{Evap}}(t)$

Where $V_{\text {Evap }}(t)$ is the total volume of water lost at time $t$, which is given by:

$V_{\text {Evap }}(t)=\frac{\text { mass of flask }(t-1)_{\text {after sampling }}-\text { mass of flask }(t)_{\text {before sampling }}}{\rho}$.

\subsection{HPLC methods}

HPLC analyses were performed using a 1200 Agilent HPLC equipped with a diode array detector. Three different HPLC methods were developed.

For carbamazepine an Eclipse XDB-CN $5 \mu \mathrm{m}(4.6 \times 150 \mathrm{~mm})$ column was used. Elution conditions were $70 \%$ of a $25 \mathrm{mM}$ ammonium acetate buffer adjusted to $\mathrm{pH} 3.0$ with formic acid and 30\% acetonitrile. The flow rate was $1.0 \mathrm{~mL} / \mathrm{min}$ and the detection wavelength was set to $220 \mathrm{~nm}$. The injection volume was $25 \mu \mathrm{L}$ and the temperature was kept at $20^{\circ} \mathrm{C}$. The total analysis time was $10 \mathrm{~min}$. Limit of detection (LOD) was $0.3 \mathrm{ppm}$ and limit of quantification (LOQ) was $1 \mathrm{ppm}$.

For sulfamethoxazole, sulfamethizole and metoprolol an Eclipse XDB-C18 $5 \mu \mathrm{m}(4.6 \times 250 \mathrm{~mm})$ column was used. The elution conditions were $80 \%$ of $20 \mathrm{mM} \mathrm{NH}_{4} \mathrm{COOH}$ buffer at $\mathrm{pH} 3.5$ (solvent $\mathrm{A}$ ) and $20 \%$ acetonitrile (solvent B) for $10 \mathrm{~min}$, then $30 \%$ of $\mathrm{B}$ ( $70 \%$ of $\mathrm{A}$ ) for $5 \mathrm{~min}$ followed by $45 \%$ of B ( $55 \%$ of A) for $5 \mathrm{~min}$. The flow rate was $0.7 \mathrm{~mL} / \mathrm{min}$ and the detection wavelength was set at $273 \mathrm{~nm}$. The injection volume was $5 \mu \mathrm{L}$ and the temperature was kept at $40{ }^{\circ} \mathrm{C}$. The total analysis time was $20 \mathrm{~min}$. LOD and LOQ were for SMX, SMZ and MTP, respectively $0.02,0.25,1 \mathrm{ppm}$ and $0.08,0.8,3 \mathrm{ppm}$.

Table 1

Percentage of pharmaceutical removal obtained with Rhodococcus rhodochrous.

\begin{tabular}{lrrrrlrr}
\hline Drug $\begin{array}{l}\text { Duration } \\
\text { (days) }\end{array}$ & \multicolumn{3}{l}{ Concentration (ppm) } & \multicolumn{2}{l}{$\begin{array}{l}\text { Biodegradation } \\
\text { (\%) }\end{array}$} \\
\cline { 2 - 6 } & Initial & Final & Corrected & $\begin{array}{l}\text { Adsorption } \\
\text { loss }\end{array}$ & $\begin{array}{l}\text { Abiotic } \\
\text { loss }\end{array}$ & \\
\hline CBZ & 28 & 9.5 & 7.6 & 7.5 & 0 & 0.6 & 15 \\
MTP & 19 & 107.8 & 132.8 & 102.9 & 0 & 4.9 & 0 \\
SMX & 36 & 31.6 & 29.3 & 14.6 & 0 & 10.6 & 20 \\
SMZ & 12 & 43.4 & 38.5 & 34.5 & 0 & 2.8 & 14 \\
TMP $^{\text {a }}$ & 7 & 92.3 & 98.1 & & & & 0 \\
\hline
\end{tabular}

a TMP correction for water loss was not done because the degradation curve was identical to the abiotic and sorption curves showing that there had been no degradation.
Table 2

Percentage of pharmaceutical removal obtained with Aspergillus niger.

\begin{tabular}{|c|c|c|c|c|c|c|c|}
\hline \multirow[t]{2}{*}{ Drug } & \multirow{2}{*}{$\begin{array}{l}\text { Duration } \\
\text { (days) }\end{array}$} & \multicolumn{5}{|c|}{ Concentration (ppm) } & \multirow{2}{*}{$\begin{array}{l}\text { Biodegradation } \\
(\%)\end{array}$} \\
\hline & & Initial & Final & Corrected & $\begin{array}{l}\text { Adsorption } \\
\text { loss }\end{array}$ & $\begin{array}{l}\text { Abiotic } \\
\text { loss }\end{array}$ & \\
\hline CBZ & 10 & 11.4 & 9.7 & 9.0 & 1.1 & 0.3 & 9 \\
\hline TMP & 14 & 91.4 & 104.7 & 92.2 & & & 0 \\
\hline
\end{tabular}

For trimethoprim an Eclipse XDB-C18 $5 \mu \mathrm{m}(4.6 \times 250 \mathrm{~mm})$ column was used. The elution conditions were $85 \%$ of methanol/ water (60:40) at pH 3.0 (solvent A) and water (solvent B) $15 \%$. The flow rate was $1.2 \mathrm{~mL} / \mathrm{min}$ and the wavelength was set at $230 \mathrm{~nm}$. The injection volume was $5 \mu \mathrm{L}$ and the temperature was kept at $35^{\circ} \mathrm{C}$. The total analysis time was $8 \mathrm{~min}$. LOD and LOQ were of 3 and $10 \mathrm{ppm}$, respectively.

\subsection{Metabolites}

Various new HPLC peaks were observed that were not detected in the control experiments. Some of these were collected using a 1200 Agilent Semi-Preparative HPLC equipped with a fraction collector. Fractions were collected with an injection volume set to $25 \mu \mathrm{L}$ in each HPLC method and then concentrated under nitrogen before further analysis.

Mass spectra analyses were performed on the collected fractions. Two different mass spectrometers were used. One was a Waters Micromass QTOF 2. The ionization was achieved by nanospray at a flow of $1 \mu \mathrm{l} / \mathrm{min}$. The recording of the spectra was done in positive mode. The energy was varied between 10 and $20 \mathrm{eV}$. The second apparatus was a Waters IonSpec 7.0T FTMS equipped with a Z-spray source. Again the positive mode was used for the analyses.

\section{Results}

\subsection{Biodegradation experiments}

Preliminary studies were performed without glucose present in the media. None of the microbes tested was able to grow with a pharmaceutical as the sole carbon source. All of the data reported, used media containing glucose.

Fig. 1 shows the effect of water evaporation on concentration of metoprolol during a biodegradation study with $R$. rhodochrous. The rising curve represents the measured data. The decreasing curve represents the same data corrected for water loss using Eqs. (1)-(4).

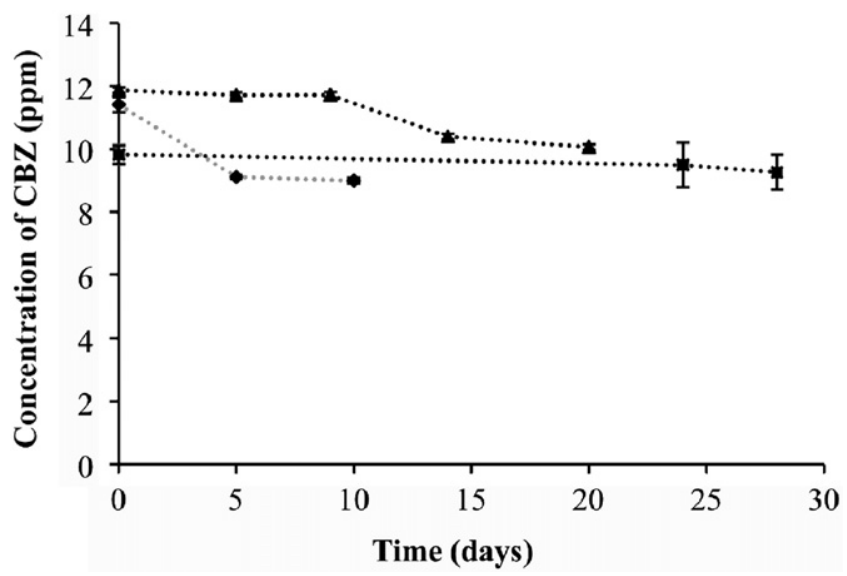

Fig. 2. Concentration of carbamazepine during biodegradation experiments ( $3 \mathrm{~g} / \mathrm{L}$ of glucose) using $(\diamond)$ Aspergillus niger, $(\boldsymbol{\Delta})$ Rhodococcus rhodochrous, and ( $\mathbf{\square})$ abiotic control (error bars: standard deviation of replicates, $<2 \%$ ). 


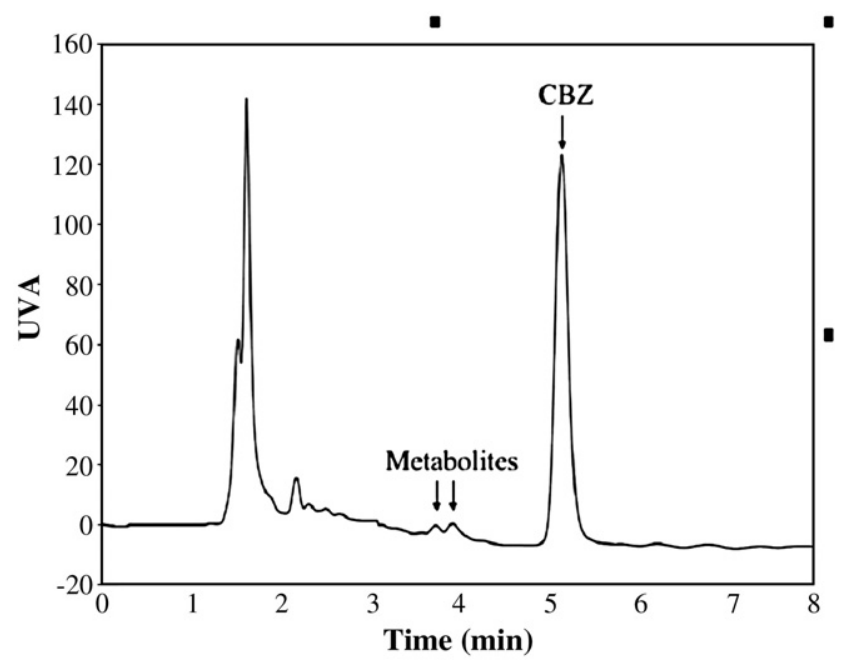

Fig. 3. Chromatogram of a sample collected after 10 days in a flask containing CBZ and Aspergillus niger.

Similar trends were observed with the measurements of the other pharmaceutical concentrations.

Tables 1 and 2 summarize the results for the biodegradation and control experiments for the various pharmaceuticals using $R$. rhodochrous and $A$. niger. These results demonstrate the importance of correcting for water loss. None of these experiments showed significant amounts of adsorption of the pharmaceuticals to the biomass. Only three pharmaceuticals, MTP, SMX and SMZ exhibited measurable amounts of abiotic degradation. In every case, the growth of the microorganism had reached the stationary phase in less than a week.

A number of other bacteria were tested in the same manner for the ability to degrade carbamazepine. None of these, which included Sphingomonas herbicidovorans, B. subtilis, P. fluorescens and P. putida, was able to degrade this compound. Nor was there any evidence for adsorption of the pharmaceutical to the biomass.

As seen in Fig. 2, A. niger was able to decrease the concentration of carbamazepine over five to ten days, which was much more rapid than the change caused by $R$. rhodochrous. However, a comparison with the control experiments using dead biomass showed that about half of this decrease was caused by adsorption to the biomass, which would explain why the change was relatively quick. Biodegradation of carbamazepine was confirmed for both of these microorgansims by

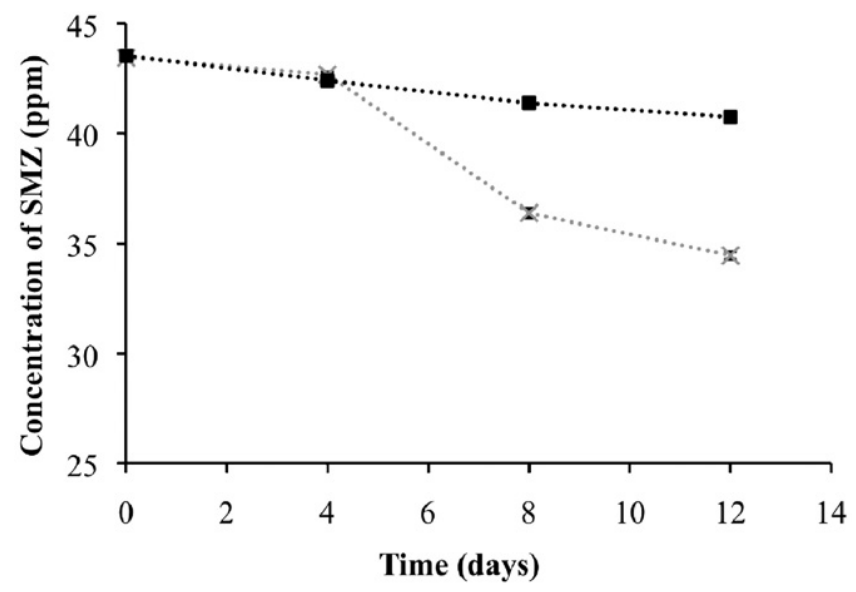

Fig. 4. Biodegradation of SMZ by Rhodococcus rhodochrous ( $3 \mathrm{~g} / \mathrm{L}$ of glucose), (x) sample, (घ) abiotic control (error bars: standard deviation of replicates, $<2 \%$ and not visible on the graph).

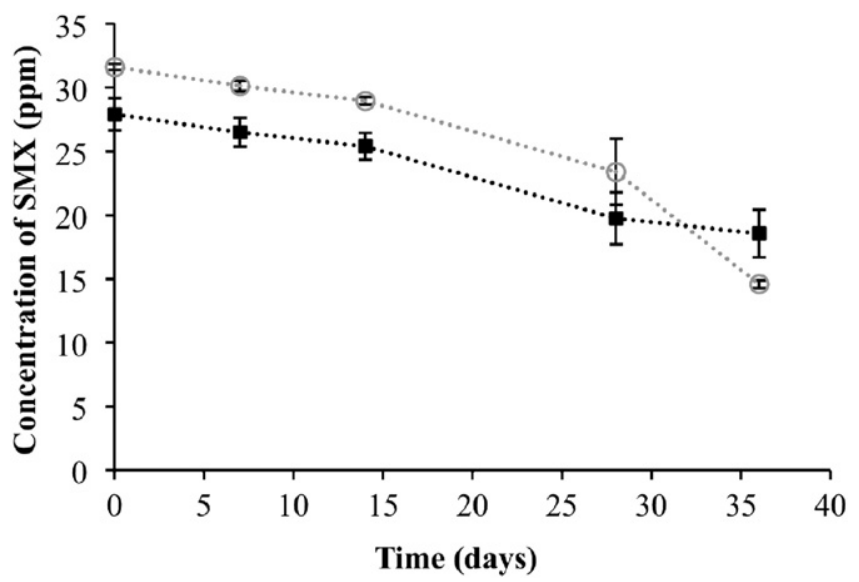

Fig. 5. Biodegradation of SMX by Rhodococcus rhodochrous ( $3 \mathrm{~g} / \mathrm{L}$ of glucose), (O) sample, abiotic control.

the observation of new peaks in the HPLC chromatograms. The two new peaks are shown in Fig. 3 for the experiment with A. niger.

Samples from the biodegradation of sulfamethizole (SMZ) and sulfamethoxazole (SMX) by $R$. rhodochrous were corrected for water loss and plotted against time in Figs. 4 and 5. The data for (SMZ) in Fig. 4 are fairly straight forward and show significant biodegradation in about ten days. Adsorption was not observed but there was some abiotic degradation (2.8\%). The results for SMX are more surprising. While there was noticeable loss over a five week period, Fig. 5 seems to show that most of this was due to abiotic degradation. However, an HPLC analysis of the spent broth showed two new peaks in the chromatogram for the flask containing $R$. rhodochrous, at 15 and 19 min but only a single new peak at 12 min in the abiotic control. An entirely different set of peaks were observed with the SMZ biodegradation experiments at 7.5, 9.5, 9.9 and $16.4 \mathrm{~min}$ but these peaks were also observed in the abiotic control experiments so these were not attributed to biodegradation. All of the metabolites observed in the abiotic controls were short lived and too unstable to identify.

For most of the metabolites observed, the low concentrations or the instability of the compounds made it impossible to attempt to characterize them. Of all of the drugs used, only the two metabolites of sulfamethoxazole (not observed in the abiotic control) were collected in sufficient quantities, by semi-preparative HPLC, to allow mass spectrum analyses. Fig. 6 presents the mass spectrum of the fraction collected at a retention time of $15 \mathrm{~min}$ in a sample from the

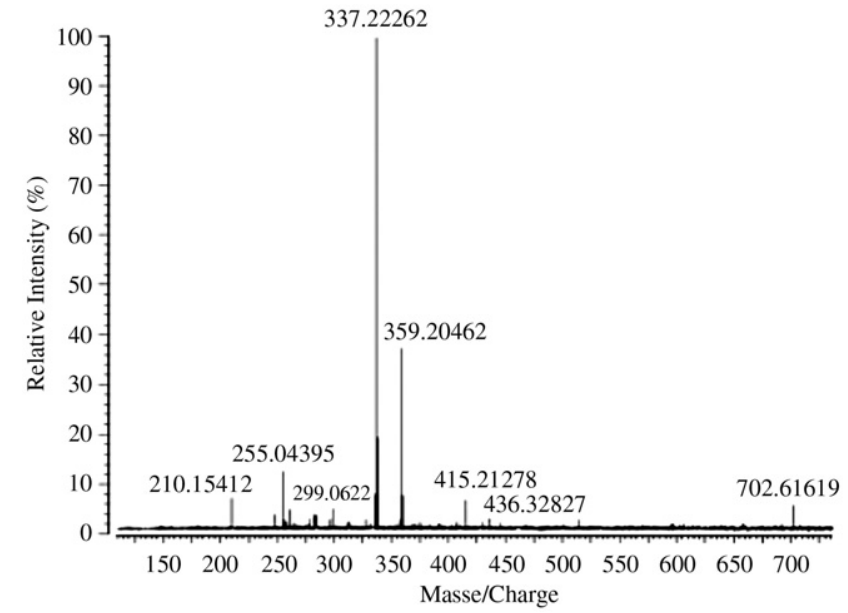

Fig. 6. Mass spectrum of SMX metabolite collected at retention time of $15 \mathrm{~min}$ (Rhodococcus rhodochrous, time $=36$ days). 
Table 3

Fragmentation observed for sulfamethoxazole and for the metabolite with $m / z$ of 255 .

\begin{tabular}{lc}
\hline Sulfamethoxazole & Metabolite with $m / z 255$ \\
\hline 92.1 & 93.1 \\
99.1 & 99.1 \\
108.1 & 109.1 \\
147.0 & 147.0 \\
156.0 & 157.0 \\
\hline
\end{tabular}

biodegradation of sulfamethoxazole by $R$. rhodochrous. The mass spectrum recorded with the IonSpec 7.0 T FTMS indicated that fraction was composed of more than one compound but most of these were in small quantity and unstable. The peak with an $m / z$ of $255(\mathrm{M}+\mathrm{H})$ was a compound that was enough stable and present in a high enough concentration to analyze further with a Micromass QTOF 2. Table 3 shows a comparison of the major peaks from the fragmentation of SMX and of this metabolite.

Mass spectrometry was also performed on a second metabolite collected from SMX biodegradation (retention time of $19 \mathrm{~min}$ ). The compound had an $m / z$ of 239.11 amu. This second metabolite was stable and resistant to fragmentation. The fragmentation pattern that was observed did not correspond to the one of SMX.

\section{Discussion}

To interpret the data it was first necessary to consider several levels of corrections and control experiments. Fig. 1 shows the results obtained for a biodegradation experiment performed over 19 days. Each point on the graph represents 5 replicates. The rising line for the raw data implies an obvious but unexpected trend of an increasing concentration of metoprolol over the 19 day period. This increase in concentration cannot be due to an increase in the pharmaceutical so there are two possible explanations of the phenomenon. There could be evaporation of water or the signal is being increased by an overlap with the signal due to some metabolite produced by the microorganism or there is a combination of both affects. However, the HPLC signal of metoprolol maintained very good symmetry and the peak of this signal, the retention time, did not vary throughout the experimentation. This indicated that a single compound was eluting at this retention time throughout the experiment.

The other line in Fig. 1 represents the concentrations corrected for water loss. On average, the loss due to evaporation measured over the duration of the experiment accounted for $7 \%$ of the initial volume of the solution contained in the shake flasks. With these corrections it can be seen that there was only a slight degradation of metoprolol over the 19 day period of the experiment. Finally, this slight decrease could be totally attributed to abiotic degradation by comparing to another type of control experiments. The overall result is that it can be concluded that metoprolol was not biodegraded in this experiment. All of the other data presented were subjected to the same analyses for water.

Another potential problem that was encountered while analyzing the data was loss of the target compound due to adsorption onto the biomass. This can be particularly important in the type of experiments<smiles>Cc1cc(NS(=O)(=O)c2ccc(O)cc2)no1</smiles>

Fig. 8. Proposed metabolite of SMX.

presented here because appreciable amounts of biomass were generated due to the presence of the easily utilized carbon source. Using control experiments with dead biomass allowed for any necessary corrections. This effect was dependent on the type of biomass being used. Adsorption was found to be significant when $A$. niger was used to degrade carbamazepine but insignificant when the experiment was done with $R$. rhodochrous. For this reason, and because none of the other bacteria tested with carbamazepine were able to cause any degradation, experiments with the rest of the pharmaceuticals were only carried out using $R$. rhodochrous.

Finally, it was necessary to eliminate effects due to abiotic degradation. This was not a problem with the experiments with carbamazepine but the experiments with both SMZ and SMX demonstrated appreciable amounts of degradation even if a microorganism was not present. The results with SMZ demonstrated that there was significantly more loss of the pharmaceutical when $R$. rhodochrous was present and growing on glucose as compared to the abiotic control experiment. This was confirmed by the presence of metabolites in the samples of broth analyzed late in the experiment that were not observed for the control experiments. Even though SMX and SMZ are similar compounds (Fig. 7) the metabolites seen were very different and none of the three metabolites observed for SMX are the same as the four observed with SMZ. The structures of the metabolites arising from the abiotic degradation were not elucidated.

Furthermore, the results with SMX are more difficult to interpret than those with SMZ. The amount of abiotic degradation for SMX is comparable to that observed in the presence of $R$. rhodochrous. However, the degradation pattern is obviously different because different metabolites were observed when the bacterium was present from the pattern observed for the abiotic control experiment. This implies that either the bacterium is competing with the abiotic degradation of SMX to generate a new type of metabolite or that it is acting on the abiotic metabolites to cause further degradation. To consider these possibilities it is necessary to characterize the metabolites from both types of degradation. Unfortunately, we have only been able to identify one metabolite.

As shown in Table 3, the MSMS of one of the metabolites from the $R$. rhodochrous experiments is very similar to the pattern of the parent SMX. The high resolution mass spectrum indicated an exact mass of $254.03612 \mathrm{amu}$ for the metabolite resulting in a less than $1 \mathrm{amu}$ difference from that of SMX, which has an exact mass of $253.05211 \mathrm{amu}$. The most probable chemical formula, considering the nitrogen rule, was $\mathrm{C}_{10} \mathrm{H}_{10} \mathrm{O}_{4} \mathrm{~N}_{2} \mathrm{~S}$. The isotopic distribution ( $\mathrm{M}+1$ and $\mathrm{M}+2$ ) obtained was consistent with the formula proposed with differences of less than $1 \%$. Based on these observations, the most<smiles>Cc1cc(NS(=O)(=O)c2ccc(N)cc2)no1</smiles>

(a)<smiles>CC1=CNN(NS(=O)(=O)c2ccc(N)cc2)S1</smiles>

(b)

Fig. 7. (a) SMX and (b) SMZ structures. 
probable compound is hydroxy-N-(5-methyl-1,2-oxazol-3-yl)benzene-1-sulfonamide, (Fig. 8). This would mean that this metabolite from the action of $R$. rhodochrous on SMX is the parent compound with the amine functional group on the aromatic ring replaced by a hydroxyl group.

This identification indicates that this metabolite is from a competing degradation reaction with abiotic degradation because it is difficult to imagine a stable abiotic intermediate in a pathway between SMX and this compound. The other biological metabolite was not identified but it is still obviously different from the abiotic metabolites and both of the $R$. rhodochrous products are more stable than any of the new compounds observed from abiotic degradation. It is interesting that there was no sign of a biological metabolite when SMZ was exposed to $R$. rhodochrous as SMZ has the same aromatic function as SMX. The fact that the amine group was not replaced with a hydroxyl function shows that the nature of the whole molecule is important for the possibility of this bioconversion to take place. The biodegradation of carbamazepine with either of two microbes also led to the accumulation of stable metabolites although in quantities that were too small for analyses. The important conclusion is that, for many of the experiments in which biodegradation occurred, metabolites were observed.

The above results show that the difficult to degrade pharmaceuticals can be much more susceptible to biodegradation if an easily used carbon source is included in the medium. The microbes used were not able to grow if one of the pharmaceuticals tested was the sole carbon source. However, with the addition of glucose to ensure significant amounts of growth, three of the target compounds could be significantly degraded if they were left in contact with the biomass for a long enough period of time. Furthermore, for two of the pharmaceuticals, SMX and CBZ, biodegradation led to the accumulation of stable metabolites and these were different and more stable than metabolites observed for abiotic degradation when it was observed.

The combination of small amounts of pharmaceuticals, microorganisms and suitable growth substrates is likely to be encountered in many parts of the environment. The removal efficiencies were found to be higher, or equivalent, for most of the drugs studied (CBZ, SMX, SMZ) to the removals reported in previous studies based on activated sludge systems (Halling-Sorensen and Ingerslev, 2000; Oppenheimer et al., 2007; Stackelberg et al., 2007). This makes these findings significant and bioconversion of pharmaceuticals must be considered as part of the analysis of environmental impact. It is particularly important to note that the metabolites from the bioconversion experiments were not observed for abiotic degradation. The biodegradation metabolites cannot be predicted from a consideration of physical and chemical degradation mechanisms.

\section{Acknowledgements}

Grateful acknowledgements are made to the Natural Sciences and Engineering Research Council of Canada (NSERC) and the Fonds québécois pour la recherche en nature et technologies (FQRNT) for their financial support.

\section{References}

Åke W, Bo G. Public health care management of water pollution with pharmaceuticals: environmental classification and analysis of pharmaceutical residues in sewage water. Drug Inf J 2005;39(3):291-7.

Daughton CG, Ternes TA. Pharmaceuticals and personal care products in the environment: agents of subtle change? Environ Health Perspect Suppl 1999;107:907-38.

Davis DL, Bradlow HL. Can environmental estrogens cause breast cancer? Sci Am 1995;273(4):166-72.

Halling-Sorensen B, Ingerslev F. Biodegradability properties of sulfonamides in activated sludge. Environ Toxicol Chem 2000;19(10):2467-73.

Jobling S, Nolan M, Tyler CR, Brighty G, Sumpter JP. Widespread sexual disruption in wild fish. Environ Sci Technol 1998;32(17):2498-506.

Jones $\mathrm{OAH}$, Voulvoulis N, Lester JN. Human pharmaceuticals in wastewater treatment processes. Crit Rev Environ Sci Technol 2005;35(4):401-27.

Kermanshahi-Pour A, Mamer OA, Cooper DG, Maric, Nicell JA. Metabolites from the biodegradation of 1.6-hexanediol dibenzoate, a potential green plasticizer, by Rhodococcus rhodochrous. J Mass Spectrom 2009;44:662-71.

Kolpin DW, Furlong ET, Meyer MT, Thurman EM, Zaugg SD, Barber LB, et al. Pharmaceuticals, hormones, and other organic wastewater contaminants in U.S. streams, 1999-2000: a national reconnaissance. Environ Sci Technol 2002;36(6):1202-11.

Nalli S, Cooper DG, Nicell JA. Interaction of metabolites with $R$. rhodochrous during the biodegradation of di-ester plasticizers. Chemoshpere 2006a;65:1510-7.

Nalli S, Cooper DG, Nicell JA. Metabolites from the biodegradation of di-ester plasticizers by Rhodococcus rhodochrous. Sci Total Environ 2006b;366:286-94.

O'Grady, D, Yargeau, V. Removal of Aqueous EE2 by Four Rhodococcus Species. Environmental Engineering Science (In print) (2009).

Oppenheimer J, Stephenson R, Burbano A, Liu L. Characterizing the passage of personal care products through wastewater treatment processes. Water Environ Res 2007;79:2564-77.

Stackelberg PE, Gibs J, Furlong ET, Meyer MT, Zaugg SD, Lippincott RL. Efficiency of conventional drinking-water-treatment processes in removal of pharmaceuticals and other organic compounds. Sci Total Environ 2007;377(2-3):255-72.

Ternes TA. Occurrence of drugs in German sewage treatment plants and rivers. Water Res 1998;32(11):3245-60.

Tixier C, Singer HP, Oellers S, Muller SR. Occurrence and fate of carbamazepine, clofibric acid, diclofenac, ibuprofen, ketoprofen, and naproxen in surface waters. Environ Sci Technol 2003;37(6):1061-8.

Yargeau V, Lopata A, Metcalfe C. Pharmaceuticals in the Yamaska River, Quebec, Canada. Water Qual Res J Can 2007;42(4):231-9.

Zwiener C. Occurrence and analysis of pharmaceuticals and their transformation products in drinking water treatment. Anal Bioanal Chem 2007;387(4):1159-62. 